\section{Les enjeux \\ économiques \\ et sociaux \\ de linfection \\ par le VIH à l'ère \\ des multithérapies \\ antirétrovirales}

Apports

des sciences humaines

et sociales

Jean-Paul Moatti, Bruno Spire jusque là dominante dans les disciplines biomédicales, qui visait à identifier sur la base d'un nombre limité de caractéristiques a priori les patients censés présenter un «haut risque» de non-observance. Elles ont montré que l'observance est un processus d'apprentissage dynamique qui dépend avant tout de l'expérience subjective vécue par les patients. La «chronicisation» de la maladie VIH a conduit les SHS à s'intéresser à l'impact des traitements sur tous les aspects de la vie quotidienne (de l'insertion professionnelle à la sexualité), en même temps qu'elles soulignent la contradiction entre les espoirs suscités par des multithérapies économiquement coût-efficaces d'une part, et la précarité sociale d'une proportion croissante de personnes atteintes, d'autre part. Ces recherches débouchent sur des recommandations visant à adapter les politiques publiques de lutte contre l'épidémie de sida tout en préservant le potentiel d'innovations qu'elles ont favorisé dans les systèmes de santé et de protection sociale, ainsi que dans les relations médecin/malade. <
Aux débuts de l'épidémie de VIH/sida, la demande adressée par les décideurs publics aux recherches en sciences humaines et sociales (SHS) visait principalement à guider les campagnes médiatiques de prévention et de promotion du préservatif. Au travers de multiples travaux sur les connaissances, attitudes,

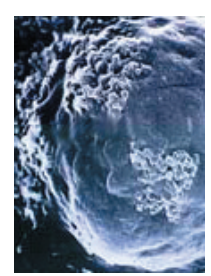

J.P. Moatti : Inserm U.379, Sciences sociales appliquées à l'innovation médicale, Institut Paoli-Calmettes, 232, boulevard de SainteMarguerite, 13273 Marseille Cedex 09, France et UFR de Sciences économiques et gestion, Université de la Méditerranée, Aix-Marseille, France.

B. Spire : Inserm U.379, Sciences sociales appliquées à l'innovation médicale,

croyances et comportements face au sida, les SHS avaient dépassé ce point de départ pour souligner que les conduites à risque de transmission du VIH ne pouvaient être appréhendées que dans le Institut Paoli-Calmettes, 232, boulevard de SainteMarguerite, 13273 Marseille Cedex 09, France et Observatoire régional de la Santé, Provence-Alpes-Côte d'Azur, France.

moatti@marseille.inserm.fr contexte global de la sexualité humaine [1], ainsi que des processus économiques, sociaux et institutionnels qui rendent certaines communautés, groupes et individus plus «vulnérables» que d'autres à l'épidémie [2]. Avec l'avènement, à partir de 1996, des multithérapies antirétrovirales (ARV), les SHS ont été sollicitées par les disciplines biomédicales pour contribuer à un meilleur suivi des prescriptions par les patients. Mais elles ont rapidement dépassé cette approche utilitariste pour s'intéresser aux effets globaux de la chronicisation de l'infection par le VIH sur la vie quotidienne des personnes atteintes, comme sur les politiques publiques de lutte contre l'épidémie $[3,4]$. 


\section{De la «prédiction» de l'observance à la dynamique de la vie quotidienne sous traitement antirétroviral}

Un niveau d'observance* très élevé $(>95 \%$ des doses prescrites), jamais obtenu précédemment dans le traitement au long cours de maladies chroniques, est d'emblée apparu comme une condition nécessaire à l'efficacité virologique des ARV [5]. Depuis, il a été confirmé qu'une bonne observance est associée à une baisse de la progression clinique vers le sida et de la mortalité chez les patients traités [6]. La non-observance, en favorisant la transmission éventuelle de souches virales résistantes aux principaux ARV, menace également l'efficacité des thérapeutiques existantes à l'échelle de la santé publique [7].

S'agissant d'un comportement qu'il est impossible d'observer en permanence, les SHS ont dissipé l'illusion que l'observance puisse être mesurée de façon objective, indépendamment du patient lui-même. Son évaluation par le prescripteur, sur la base du colloque singulier médecin/malade, s'avère peu fiable [8]. Les mesures biologiques de charge virale et les dosages pharmacologiques d'antirétroviraux peuvent contribuer à valider les déclarations des patients, mais ne constituent pas en soi une mesure d'observance; il a d'ailleurs été montré qu'elles s'avèrent moins sensibles que des questionnaires administrés aux patients pour détecter les comportements de non-observance [9]. Le comptage des comprimés par la pharmacie délivrant les médicaments et le recours aux piluliers électroniques ont démontré leur fiabilité dans des études expérimentales, mais peuvent difficilement être étendus en routine à un grand nombre de patients [10]. En définitive, en dépit d'indéniables biais de mémorisation ou de conformisme, les méthodes d'interrogation directe des patients par auto-questionnaires, mises au point par les SHS, ont fait la preuve de leur validité pour suivre l'observance au cours du temps [11].

Ce suivi longitudinal a permis de remettre en cause l'approche prédictive de l'observance, jusque-là dominante dans la littérature biomédicale [12] et dans les premières études transversales auprès de patients sous multithérapies [13]: cette approche visait à identifier, à partir d'un nombre limité de caractéristiques psychologiques et socio-démographiques, les patients à haut risque de non-observance, afin de les inciter à modifier leurs comportements ou de subordonner la décision

* L'observance est définie comme le respect des prescriptions médicales dans la prise et le suivi de son traitement par le patient. d'initier le traitement à la garantie d'une meilleure observance. Dans la cohorte française APROCO, qui depuis mai 1997 suit des patients infectés par le VIH-1 commençant une multithérapie comprenant au moins un inhibiteur de protéase, quelques caractéristiques d'inclusion (notamment un âge jeune, un logement précaire, l'absence de soutien de l'entourage, une consommation d'alcool élevée et une médiocre qualité de la relation avec le médecin prescripteur) sont effectivement associées, dans les analyses multivariées, à la non-observance du traitement quatre mois plus tard. Mais celle-ci est bien mieux expliquée si les évolutions intervenues du fait de l'expérience subjective par les patients de ces quatre premiers mois de traitement sont prises en compte: une détérioration du score psychométrique de dépression, de la perception subjective de l'état de santé et de la confiance dans l'efficacité du traitement, d'une part, et une augmentation de la perception de la toxicité des traitements et de la consommation d'alcool ou de tabac, d'autre part sont associées, à une moindre observance [14]. La cohorte APROCO, et son équivalent italien (cohorte ICONA), ont montré le rôle de la perception subjective des effets secondaires (la fatigue, les diarrhées et nausées étant les effets les plus fréquemment rapportés) comme facteur de non-observance, cette perception pouvant s'écarter notablement de la toxicité médicalement relevée $[14,15]$. De même, les patients initialement observants au cours des premiers mois présentent un risque accru de rupture ultérieure d'observance s'ils déclarent une altération de leur image corporelle qu'ils attribuent aux lipodystrophies [16].

En complétant le suivi clinique et biologique de cohortes par le recueil de données socio-comportementales, les SHS ont révélé le caractère dynamique, impossible à prédire lors de l'initiation du traitement, des comportements d'observance. Dans la cohorte APROCO, les proportions de patients déclarant une observance totale ( $100 \%$ des doses prescrites), modérée ( $80 \%$ à $99 \%$ ) ou basse ( $<80 \%)$ dans les quatre jours précédents sont quasi constantes à chaque visite des 20 premiers mois de suivi (respectivement $60 \%, 30 \%$ et $10 \%$ des patients). Mais cette apparente stabilité transversale de l'observance dissimule des fluctuations au cours du temps: seuls $31 \%$ des patients maintiennent une observance totale à tous les suivis, alors que la majorité d'entre eux (52\%) présentent occasionnellement des épisodes de non-observance, et que les $17 \%$ restants ne sont jamais totalement observants [17].

L'observance est bien un processus d'apprentissage, dont les rythmes peuvent différer selon les groupes d'appartenance et les caractéristiques individuelles des 
patients, et qui demeure sensible en continu à l'influence de l'environnement personnel, familial et social, ainsi qu'à l'expérience vécue du traitement luimême et à son impact sur la qualité de vie. Si l'accès aux multithérapies s'est logiquement accompagné d'une amélioration globale de la qualité de vie des personnes atteintes, telle qu'elle peut être mesurée sur différentes échelles psychométriques, certaines de ses dimensions (celles relevant de la fatigue ou de la douleur physique, notamment) peuvent se détériorer au cours du suivi [18-20]. De plus, certains patients bénéficient, moins que d'autres, de gains en qualité de vie: c'est «paradoxalement » le cas, un an après l'initiation du traitement dans la cohorte APROCO, de ceux qui maintiennent une activité professionnelle [20].

La remise en cause de l'approche prédictive de l'observance a favorisé un regard critique sur les stéréotypes qui lui étaient associés par une partie des professionnels de santé, comme l'idée d'une moindre observance intrinsèque chez les toxicomanes [21]. De tels jugements émis a priori sur les capacités d'observance ont pu expliquer le retard relatif dans la mise sous traitement antirétroviral des patients toxicomanes, en comparaison d'autres groupes à caractéristiques cliniques et immunologiques identiques, même dans des systèmes de santé comme le nôtre où n'existent pas de barrières financières pour l'accès au traitement [22]. Pourtant, dans la cohorte française MANIF 2000 de patients infectés par voie toxicomane, ceux qui bénéficient en parallèle d'un traitement de substitution aux opiacés par buprénorphine apparaissent autant, voire plus, observants de leur multithérapie antirétrovirale que ceux qui ont renoncé à toute toxicomanie, et les niveaux d'observance dans cette cohorte sont équivalents à ceux observés par ailleurs avec les patients d'autres groupes de transmission [23].

Les recherches en SHS ont contribué au développement d'une approche alternative de l'observance que l'on peut qualifier d'empathique, puisqu'elle s'efforce d'aider chaque patient à résoudre ses difficultés de prise de médicaments à partir de son expérience propre. Cette approche est désormais intégrée, du moins en France, dans les recommandations cliniques de prise en charge [24]. Elle inspire la plupart des expérimentations randomisées en cours visant à définir les modalités les mieux appropriées d'interventions psycho-socio-comportementales pour améliorer l'observance aux différentes étapes du traitement [25], comme les consultations d'observance qui se créent dans un nombre croissant de services cliniques, ou les interventions de soutien par les pairs mises en place par les associations.
L'adaptation actuelle des stratégies thérapeutiques nécessite une poursuite de ces recherches élargies aux divers aspects de la vie quotidienne des patients. Par exemple, rien ne permet de prédire l'impact des stratégies, en cours d'essais cliniques, d'interruptions thérapeutiques programmées, comme celui des interruptions de fait chez des patients en succès immuno-virologique qui souhaitent un arrêt momentané: si les interruptions, en réduisant la fatigue et la toxicité associées aux ARV, peuvent sembler favorables à une amélioration de l'observance lors de la reprise, il existe aussi de forts arguments (perte des routines qui facilitaient le suivi des prescriptions, complexité des protocoles alternant les phases de traitement et d'arrêt) en faveur de l'hypothèse inverse de risques accrus de non-observance [12].

\section{De l'accès aux soins à la prise en compte de la vulnérabilité économique et sociale}

En France, la grande majorité des personnes infectées (dont la prévalence est estimée entre 70000 et 127000 cas) bénéficie d'un accès gratuit aux soins: le nombre de personnes suivies pour une prise en charge hospitalière du VIH était estimé de 54000 à 70000 pour l'année 1999, et la proportion des patients sous ARV parmi les patients suivis est désormais stable, autour de $87 \%$ en 2001 [24]. Contrairement aux violentes controverses qu'elle suscite pour les pays en développement, la faisabilité économique des multithérapies n'a jamais semblé poser de problème dans les pays développés. Leur diffusion à grande échelle a précédé l'évaluation de leur impact sur les coûts et sur les ratios coût-efficacité de la prise en charge du VIH. Il est vrai que, dans les pays développés, les coûts médicaux directs de prise en charge des patients infectés par le VIH (estimés par exemple à un total de 6,7 à 7,8 milliards de dollars aux États-Unis en 1996) ne représentent qu'une part infime (moins de $0,1 \%$ ) du total des dépenses de santé [26].

De plus, il est rapidement apparu que les surcoûts induits par les prix unitaires élevés des molécules antirétrovirales et par le suivi biologique des patients sous traitement ARV étaient, en tout ou partie, compensés par les économies liées à la réduction des hospitalisations pour épisodes d'infections opportunistes et à la possibilité d'arrêter les prophylaxies, souvent coûteuses, de ces infections opportunistes chez les patients sous ARV $[27,28]$. Il est probable qu'avec l'allongement de l'espérance de vie permis par les multithérapies et l'accroissement du nombre des patients en échec thérapeutique «sévère», les coûts médicaux directs finissent par augmenter, dès lors qu'on raisonne 
sur le cycle de vie complet de prise en charge de cohortes de patients [29]. Mais, de toute façon, il faut se souvenir que les stratégies médicales habituellement considérées comme coût-efficaces ne sont pas uniquement celles qui se traduisent par des surcoûts nuls, voire des économies nettes pour les dépenses de santé. Il est classique de considérer comme particulièrement coût-efficaces, et donc devant être adoptées sans discussion, des innovations biomédicales qui permettent le gain d'une année de vie supplémentaire pour un surcoût de dépenses de santé qui demeure inférieur à deux fois le Produit national brut annuel par tête (l'idée étant que, à ce niveau, on peut être quasiment certain que ce coût additionnel sera plus que compensé par les bénéfices obtenus en termes de productivité économique, et plus largement de bien-être, liés à cette réduction de la mortalité) [30]. Les multithérapies antirétrovirales satisfont tout à fait un tel critère dans les pays de l'OCDE où deux fois le PNB par tête équivaut environ à 50000 US \$, puisque leur coût additionnel par année de vie (ajustée sur la qualité de vie) supplémentaire gagnée est de l'ordre de 13000 à 26000 US \$ [27, $31]$, et se compare favorablement avec les ratios coût par année de vie gagnée de la prophylaxie des infections opportunistes ainsi que de nombreuses interventions de prévention du VIH [31].

Si l'accès aux soins et aux ARV varie en fonction des caractéristiques générales des systèmes d'assurancemaladie des différents pays développés, une tendance lourde, actuellement observée partout, est l'accentuation de la précarité sociale parmi les personnes atteintes. D'une part, les nouveaux diagnostics de séropositivité interviennent de plus en plus souvent dans les catégories les plus défavorisées, à la fois parce que ce sont dans ces catégories que le recours tardif au dépistage, à un stade déjà avancé d'immunodéficience, demeure fréquent [32], et parce que l'incidence des nouvelles infections tend à se concentrer dans les groupes les plus vulnérables (comme chez les femmes des minorités ethniques aux États-Unis, par exemple) $[2,33]$. D'autre part, la chronicisation de leur maladie affecte la trajectoire sociale et professionnelle des personnes atteintes et s'accompagne souvent d'un appauvrissement de leurs ressources [3, 4]. En 1997, on estimait en Australie qu'un tiers des personnes infectées vivaient en dessous du seuil de pauvreté [34]. L'enquête réalisée en 2001 auprès d'un échantillon de 900 patients infectés par le VIH, représentatifs de la file active des services hospitaliers des régions $1 / e-d e-$ France et Provence-Alpes-Côte d'Azur, confirme leur situation «socialement défavorisée» par rapport à la moyenne de la population française, et souligne que plus de $30 \%$ d'entre eux tirent l'essentiel de leurs ressources des prestations sociales liées à la reconnaissance d'un statut de personne handicapée [35]. La bonne insertion sociale de la majorité des patients contaminés par voie homo- ou bisexuelle (environ un tiers de l'échantillon, dont la moitié ont un niveau d'études supérieur au bac et les deux tiers occupent un emploi) contraste, cependant, avec la précarité sociale de la grande majorité des patients contaminés par injection de drogues (un quart de l'échantillon, deux sur trois sans emploi et ayant arrêté l'école au niveau primaire ou au collège, $80 \%$ co-infectés par le VHC). Le groupe des patients contaminés par voie hétérosexuelle (un peu plus du tiers de l'échantillon) présente des situations sociales plus diversifiées (un sur deux a un emploi), mais les femmes, en particulier étrangères, y constituent un sous-ensemble plus particulièrement marqué par la précarité. Même lorsqu'un accès gratuit aux ARV est garanti, la précarité demeure synonyme d'un moins bon pronostic de survie [36].

Alors que les progrès thérapeutiques alimentent chez les personnes atteintes des aspirations légitimes à la reprise de la vie professionnelle et affective, comme du désir d'enfant, la précarisation économique et sociale d'un nombre croissant d'entre elles limite leurs capacités à se projeter de nouveau dans le futur. En dépit des dispositifs réglementaires destinés à favoriser le maintien des personnes malades dans l'entreprise (droits au congé de maladie, mi-temps thérapeutique, droits des travailleurs handicapés), la sauvegarde, l'accès ou le retour à l'emploi semblent plutôt dépendre d'arrangements informels qui permettent de garder secrète la séropositivité vis-àvis de tout ou partie du milieu de travail [37]. La persistance de discriminations dans l'accès aux contrats d'assurance, comme aux crédits à la consommation ou à la propriété immobilière, peuvent s'opposer aux projets de constitution d'un patrimoine [35].

Les multithérapies, qui peuvent à la fois redonner le désir et la forme physique pour réengager une vie sexuelle active, mais aussi provoquer certains troubles des fonctions sexuelles [38], ont le mérite de faire sortir la question de la sexualité des personnes atteintes du déni qu'elle subissait depuis les débuts de l'épidémie [39]. Leur diffusion suscite cependant l'inquiétude que la disponibilité de thérapeutiques efficaces favorise la recrudescence des comportements à risque, en alimentant un sentiment de fausse sécurité dans la majorité séronégative de la population, et en favorisant la reprise d'une activité sexuelle non protégée dans la population séropositive accédant aux ARV [40]. De nombreuses enquêtes ont bien mis en lumière une telle recrudescence, tant parmi les homo- et bisexuels mas- 
culins [41, 42] qu'en population générale [43], dans la période qui a suivi la diffusion des multithérapies. La surveillance épidémiologique atteste également d'une remontée de l'incidence des maladies sexuellement transmissibles [44]. Au plan des attitudes et des croyances, il est possible d'identifier des corrélations entre l'«optimisme» entretenu par l'existence des multithérapies et un moindre intérêt pour la prévention du VIH dans certains groupes $[42,45]$. En revanche, sur le plan des comportements à risque, il est difficile de faire la part de ce qui tient effectivement à l'impact des traitements et de ce qui relève plutôt d'effets de générations ou de rendements décroissants, sur la longue durée, des efforts préventifs. Quant aux conséquences de l'accès aux multithérapies sur la baisse ou l'augmentation des comportements sexuels à risque chez les personnes déjà atteintes, l'évidence actuelle demeure contradictoire $[46,47]$.

En fait, l'impact de la diffusion des traitements sur la dynamique de transmission du VIH est difficile à prédire. D'une part, il est démontré que la baisse de la charge virale réduit la probabilité de transmission du VIH lors de chaque rapport sexuel. D'autre part, cet effet favorable à une réduction de l'incidence peut être contrecarré si l'allongement de l'espérance de vie des personnes atteintes se traduit par une augmentation du nombre d'interactions sexuelles non protégées entre partenaires sérodiscordants [48]. En définitive, c'est bien de l'évolution des comportements des personnes atteintes, et de l'efficacité de la prévention secondaire en leur direction, que dépend la dynamique future de l'épidémie dans nos pays [40].

\section{De l'exceptionnalisme à la normalisation?}

Les politologues ont souligné l'«exceptionnalisme» des politiques de lutte contre le VIH/Sida mises en place à l'origine, dans la plupart des pays développés dont la France, en ce qu'elles introduisaient une rupture radicale avec les pratiques historiquement antérieures de contrôle des épidémies [49]. Le souci légitime de réduire les discriminations dont le fonctionnement social spontané menaçait les personnes infectées est au cœur de ces politiques. II a parfois conduit (par exemple, en bannissant la notion de «groupe à risque» jugée porteuse d'un danger de stigmatisation) à une prudence excessive en matière d'interventions ciblées sur les groupes les plus vulnérables ou de prévention secondaire chez les personnes déjà atteintes [4]. Mais, en construisant des dispositifs spécialisés sur la prévention et la prise en charge du VIH, en privilégiant l'incitation aux changements de comportements plutôt que la contrainte et en s'appuyant sur une mobilisation sans précédent des acteurs, à commencer par les malades eux-mêmes et leurs associations de soutien, la lutte contre le sida a introduit de nombreuses innovations tant institutionnelles que culturelles.

$\varepsilon n$ France, la généralisation des dossiers médicaux informatisés, la constitution de réseaux médecine de villehôpital, la création d'appartements thérapeutiques, l'introduction des politiques de réduction des risques et des dommages comme des traitements de substitution aux opiacés pour les toxicomanes, l'amélioration des soins palliatifs, la reconnaissance législative des droits des patients et l'évolution vers un modèle de décision thérapeutique partagée entre clinicien et patient, comme de nombreux autres domaines du système de santé et de protection sociale, ont bénéficié des avancées acquises par et dans la lutte contre le Sida.

Les progrès thérapeutiques des sept dernières années ont alimenté l'idée d'une nécessaire «normalisation» de ces politiques publiques de lutte contre l'épidémie $[49,50]$. Les recherches en SHS mettent effectivement en lumière des situations où une meilleure intégration de la prise en charge du VIH au fonctionnement «normal» de l'ensemble du système de soins et d'aide sociale ne peut que se révéler bénéfique. Parmi les professionnels du santé, il a été montré l'importance des facteurs extra-médicaux, liés à une plus ou moins grande proximité personnelle et culturelle avec les patients traités, dans la variabilité des attitudes de prise en charge du VIH [51]. C'est dire la nécessité d'efforts nouveaux de coordination et d'homogénéisation face à la diversification des catégories de professionnels désormais concernés par le suivi des patients sous ARV: gastro-entérologues et hépatologues pour la co-infection par le VHC (virus de l'hépatite C), oncologues, psychologues et psychiatres (le suivi psychologique des personnes atteintes demeurant notoirement inadapté [52]), aides à domicile pour lesquelles subsistent de nombreux besoins non satisfaits [35]. De même, la moindre part des hospitalisations traditionnelles dans la prise en charge implique d'inévitables réallocations des ressources pour éviter des gaspillages et promouvoir des économies d'échelle.

Mais les recherches en SHS démontrent également que l'intégration de la lutte contre le Sida dans les dispositifs existants n'a de sens que si elle garantit et étend les avancées qu'elle a justement favorisées. En France, le VIH est la première pathologie à avoir justifié la mise en place d'une agence de recherches qui lui soit spécifiquement dédiée (I'ANRS), et les succès obtenus, entre autres dans les recherches en SHS, valident ce choix qui devrait aujourd'hui servir de modèle pour d'autres problèmes importants de santé publique, comme le cancer. $\diamond$ 


\section{SUMMARY}

Economic and socio-behavioural issues related to highly active antiretroviral therapies

for HIV infection: the contribution of social science research

This article presents a synthesis of the main topics covered by social science research (SSR) on HIV/AIDS, since the advent of highly active antiretroviral therapies (HAART) in 1996. SSR has shown that non-adherence cannot be reliably «predicted» on the sole basis of a few a priori patient characteristics that clinicians could easily identify before initiation of HAART, and that a dynamic approach to adherence, continuously monitoring the impact of patients' subjective experience with HAART is needed. In relationship with the evolution of HIV infection toward a «chronic disease», SSR has dealt with the impact of HAART on all aspects of patients' daily lives (from employment and professional status to sexuality). It has also emphasised the potential contradictions between the hopes generated by these costeffective therapeutic advances, on the one hand, and the high social vulnerability of a growing proportion of people living with HIV-AIDS, on the other hand. Finally, SSR suggests recommendations for «normalising AIDS public policies without losing the potential for innovations that the fight against this epidemic has introduced in health care and «Social Security» systems, as well as physician-patient's relationships. $\diamond$

\section{RÉFÉRENCES}

1. Bajos N, Bozon M, Ferrand A, Giami A, Spira A et le groupe ACSF. La sexualité aux temps du Sida. Paris: PUF, 1998.

2. Delor F, Hubert M. Revisiting the concept of vulnerability. Soc Sci Med 2000; 50: 1557-70.

3. Pierret J. Everyday life with AIDS/HIV : surveys in the social sciences. Soc Sci Med 2000;

50: 1589-98.

4. Moatti JP, Souteyrand Y. HIV/AIDS social and behavioural research: past advances and thoughts about the future. Soc Sci Med 2000; 50: 1519-32.
5. Paterson DL, Swindells S, MohrJ, et al. Adherence to protease inhibitor therapy and outcomes in patients with HIV infection. Ann Intern Med 2000; 133: 21-30.

6. Bangsberg DR, Perry $S$, Charlebois ED, et al. Nonadherence to highly active antiretroviral therapy predicts progression to AIDS. AIDS 2001 ; 15: 1181-3.

7. Wainberg M, Friedland G. Public health implications of antiretroviral therapy and HIV drug resistance. JAMA 1998; 279: 1977-83.

8. Bangsberg DR, Hecht FM, Clague $\mathrm{H}$, et al. Provider assessment of adherence to HIV antiretroviral therapy. J Acquir Immune Defic Syndr 2001; 26: 435-42.
9. Duran S, Solas C, Spire B, et al. Do HIV-infected injecting drug users overreport adherence to highly active antiretroviral therapy? A comparison between patients' selfreports and serum protease inhibitor concentrations in the French Manif 2000 cohort study. AIDS 2001 ; 15: 1075-7.

10. Wendel CS, Mohler MJ, Kroesen K, Ampel NM, Gifford AL, Coons SJ. Barriers to use of electronic adherence monitoring in an HIV clinic. Ann Pharmacother 2001; 35: 1010-5.

11. Chesney MA, Ickovics JR, Chambers DB, et al. Selfreported adherence to antiretroviral medications among participants in HIV clinical trials: the AIDS clinical trials group (AACTG) adherence instruments. AIDS Care 2000; 12: 255-66.

12. Moatti JP, Spire B, Duran S. Un bilan des recherches socio-comportementales sur l'observance des traitements dans I'infection à VIH: au delà des modèles biomédicaux? Rev Epidemiol Santé Publ 2000; 48: 182-9.

13. Gordillo V, del Amo J, Soriano V, González-Lahoz J. Sociodemographic and psychological variables influencing adherence to antiretroviral therapy. AIDS 1999; 13: 1763-9.

14. Spire B, Duran S, Souville M, Leport C, Raffi F, Moatti JP. Adherence to highly active antiretroviral therapies (HAART) in HIVinfected patients: from a predictive to a dynamic approach. Soc Sci Med 2002; 54: 1481-96.

15. Ammassari A, Murri R, Pezzotti P, et al. Selfreported symptoms and medication side effects influence adherence to highly active antiretroviral therapy in persons with HIV infection. J Acquir Immune Defic Syndr 2001; 28: 445-9.
16. Duran $S$, Savès $M$, Spire B, et al. Failure to maintain long-term adherence to highly active antiretroviral therapy: the role of lipodystrophy. AIDS 2001; 15: 2441-4.

17. Carrieri P, Cailleton V, Le Moing $V$, et al. The dynamic of adherence to highly active antiretroviral therapy: results from the French national APROCO cohort. J Acquir Immune Defic Syndr 2001 ; 28: 232-9.

18. Nieuwkerk PT, Gisolf $\varepsilon H$, Reijers $M H$, et al. Longterm quality of life outcomes in three antiretroviral treatment strategies for HIV-1 infection. AIDS 2001; 15: 1985-91.

19. Low-Beer S, Chan K, Wood $\varepsilon$, et al. Health related quality of life among persons with HIV after the use of protease inhibitors. Qual Life Res 2000; 9: 941-9.

20. Carrieri MP, Spire B, Duran $S$, et al. Health-related quality of life after one year of highly active antiretroviral therapy. J Acquir Immune Defic Syndr 2003; 32: 38-47.

21. Strathdee SA, Palepu A, Cornelisse PG, et al. Barriers to use of free antiretroviral therapy in injection drug users. JAMA 1998; $280: 547-9$

22. Carrieri MP, Moatti JP, Vlahov D, et al. Access to antiretroviral treatment among French HIV-infected injection drug users (IDUs); the influence of continued drug use. J Epidemiol Community Health 1999; 53: 4-8.

23. Moatti JP, Carrieri MP, Spire B, Gastaut JA, Cassuto JP, Moreau J. Adherence to HAART in French HIVinfected injecting drug users: the contribution of buprenorphine drug maintenance treatment. The Manif 2000 study group. AIDS 2000; $14: 151-5$. 
24. Delfraissy JF. Prise en charge des personnes infectées par le VIH. Recommandations du groupe d'experts. Rapport 2002. Paris: MédecineSciences/Flammarion, 2002.

25. Tuldra A, Fumaz CR, Ferrer MJ, et al. Prospective randomized two-arm controlled study to determine the efficacy of a specific intervention to improve long-term adherence to highly active antiretroviral therapy. $J$ Acquir Immune Defic Syndr $2000 ; 25: 221-8$.

26. Hellinger FJ, Fleishman J A. Estimating the national cost of treating people with HIV disease: patient, payer, and provider data. J Acquir Immune Defic Syndr 2000; 24 : 182-8.

27. Sendi PP, Bucher HC, Harr T, et al. Cost effectiveness of highly active antiretroviral therapy in HIV-infected patients. Swiss HIV cohort study. AIDS 1999 ; 13: 1115-22.

28. Bozzette SA, Joyce G, McCaffrey DF, et al. Expenditures for the care of HIV-infected patients in the era of highly active antiretroviral therapy. HIV cost and services utilization study consortium. N Engl J Med 2001; 344: 817-23.

29. Keiser $P$, Nassar $N$, Kvanli MB, Turner D, Smith JW, Skiest D. Long-term impact of highly active antiretroviral therapy on HIV-related health care costs. J Acquir Immune Defic Syndr 2001 ; 27 : 14-9.

30. Garber A, Phelps C $\varepsilon$. Economic foundations of cost-effectiveness analysis. J Health Eco 1997; 16: 1-31.
31. Freedberg KA, Losina $\varepsilon$, Weinstein MC, et al. The cost effectiveness of combination antiretroviral therapy for HIV disease. $N$ Engl J Med 2001; 344: 824-31.

32. Vernay-Vaisse C, Enel P, Ben Diane MK, Rey D, Carrieri MP, Obadia Y. Facteurs associés à la découverte de la séropositivité au VIH à un stade d'immunodépression avancé. BEH 2002; 15: 61-3.

33. Karon JM, Fleming PL, Steketee RW, De Cock KM. HIV in the United States at the turn of the century: an epidemic in transition. Am J Publ Health 2001; 91: 1060-8.

34. Ezzy D, De Visser R, Bartos M. Poverty, disease progression and employment among people living with HIV/AIDS in Australia. AIDS Care 1999; 11: 405-14.

35. Obadia Y, Marimoutou C, Bouhnik AD, Lert F, DraySpira R, Bousquet F. Les aides apportées aux personnes atteintes par I'infection à VIH-SIDA: une enquête auprès des patients des services de soins en Île-de-France et en Provence-Alpes-Côte d'Azur. Etudes et Résultats. Paris: DREES, 2002; n²03.

36. Piketty C, Castiel P, Giral P, et al. Lack of legal income is strongly associated with an increased risk of AIDS and death in HIV-infected injecting drug users. AIDS Care 1999; 11 : 429-36.

37. Fabre G, Tchobanian S. Les tensions actuelles entre le VIH et l'emploi. Sciences Sociales et Santé 2001; 19: 43-66.

38. Colson AE, Keller Marla J, Sax PE, Pettus PT, Platt R, Choo PW. Male sexual dysfunction associated with antiretroviral therapy. J Acquir Immune Defic Syndr 2002; 30 : 27-32.
39.Schiltz MA, Sandfort TG. HIVpositive people, risk and sexual behaviour. Soc Sci Med 2000; 50: 1571-88.

40. Lert F. Advances in HIV treatment and prevention: should treatment optimism lead to prevention pessimism ? AIDS Care 2000; 12: 745-55.

41. Dukers NH, Spaargaren J, Geskus RB, Beijnen J, Coutinho RA, Fennema HS. HIV incidence on the increase among homosexual men attending an Amsterdam sexually transmitted disease clinic: using a novel approach for detecting recent infections. AIDS 2002; 16: 19-24.

42. Adam P, Delmas MC, Brunet JB. Impact des nouveaux traitements antirétroviraux sur les attitudes et les comportements préventifs des lecteurs de la presse gay. De l'impact perçu par les individus à la mesure d'une influence effective. In: Lert F, Souteyrand y, eds. Séropositivité, vie sexuelle et risque de transmission du VIH. Collection Sciences Sociales et Sida, Paris: Agence Nationale de Recherche sur le Sida. 1999: 43-53.

43. Johnson AM, Mercer $\mathrm{CH}$, Erens B, et al. Sexual behaviour in Britain: partnerships, practices, and HIV risk behaviours. Lancet 2001; 358: 1835-42.

44. Do AN, Hanson DL, Dworkin MS, Jones JL. Risk factors for and trends in gonorrhea incidence among persons infected with HIV in the United States. AIDS 2001; 15: 1149-55.

45. Van de Ven P, Kippax S, Knox S, Prestage G, Crawford J. HIV treatments optimism and sexual behaviour among gay men in Sydney and Melbourne. AIDS 1999; 13: 2289-94.
46. Scheer S, Chu PL, Klausner JD, Katz MH, Schwarcz SK. Effect of highly active antiretroviral therapy on diagnoses of sexually transmitted diseases in people with AIDS. Lancet 2001; 357: 432-5.

47. Bouhnik AD, Moatti JP, Vlahov D, et al. Highly active antiretroviral treatment does not increase sexual risk behaviour among French HIV infected injecting drug users. J Epidemiol Commun Health $2002 ; 56$ : 349-53.

48. Law MG, Prestage $G$, Grulich A, Van de Ven P, Kippax S. Modelling the effect of combination antiretroviral treatments on HIV incidence. AIDS 2000; 15: 1287-94.

49. Bayer R. Clinical progress and the future of HIV exceptionalism. Arch Intern Med 1999; 159: 1042-8.

50. Rosenbrock R, DuboisArber F, Moers M, Pinell P, Schaeffer D, Setbon M. The normalization of AIDS in Western European countries. Soc Sci Med 2000; 50: 1607-29.

51. Landman R, Moatti JP, Perrin V, Huard P and the PAMPA Study Group. Variability of attitudes toward early initiation of HAART for HIV infection: a study of French prescribing physicians. AIDS Care 2000; 12: 711-6.

52. Ickovics JR, Hamburger $M E$, Vlahov D, et al. Mortality, CD4 cell count decline, and depressive symptoms among HIV-seropositive women: longitudinal analysis from the HIV epidemiology research study. JAMA 2001; 285: 1466-74.

\section{TIRÉS À PART}

J.P. Moatti 